\title{
La Perception Du Risque Routier. Le Cas De La Conduite Des Conducteurs Professionnels De Transport En Commun A Abidjan
}

\author{
Tieffi Hassan Guy Roger, (Dr) \\ Kanga Kouakou Bruno, (Dr) \\ Achi Essétchi Narcisse, (Dr)
}

Centre Ivoirien d'Etude et de Recherche en Psychologie Appliquée (CIERPA), Université Felix Houphouet-Boigny d'Abidjan, Côte d'Ivoire

Doi: 10.19044/esj.2017.v13n32p142 URL:http://dx.doi.org/10.19044/esj.2017.v13n32p142

\begin{abstract}
This study examines the perception of the risk of accidents related to the driving activity of the Public Transport Professional Drivers (CPTC) in Abidjan. 186 CPTC of Abidjan, whose age varies between 18 and 50 years, and who holds a different driving license are investigated. Their perception of risk is measured from a questionnaire consisting of 37 items on a 5-point scale. The results indicate that there are as many CPTCs that perceive the least risk of driving-related accidents as those who perceive it the most. They also show that the age and duration of driving licenses influence the perception of the risk of CPTC accidents.
\end{abstract}

Keywords: Perception, risk, professional drivers, accidents, Ivorian context

\section{Résumé}

La présente étude examine la perception du risque d'accidents liés à l'activité de conduite automobile des Conducteurs Professionnels de Transport en Commun (CPTC) à Abidjan. 186 CPTC d'Abidjan dont l'âge varie entre 18 et 50 ans, et ayant une durée de détention du permis de conduire différente sont enquêtés. Leur perception du risque est mesurée à partir d'un questionnaire composé de 37 items sur une échelle à 5 points. Les résultats indiquent qu'il existe autant de CPTC qui perçoivent le moins le risque d'accidents liés à la conduite que ceux qui le perçoivent le plus. Ils montrent aussi que, l'âge et la durée de détention du permis de conduire influencent la perception du risque d'accidents des CPTC.

Mots-clés: Perception, risque, conducteurs professionnels, accidents, contexte ivoirien 


\section{Introduction}

Certains ouvrages spécialisés d'histoire contemporaine nous révèlent que l'invention de l'automobile a contribué aux villes de s'étendre et de réduire les temps de parcours d'une agglomération à une autre. Autrefois réservée à une catégorie de personnes socialement aisées, l'automobile a dépassé le cadre du luxe pour être maintenant une nécessité. Les industriels ont donc compris qu'il faille produire plus de véhicules de transport pour satisfaire la demande. C'est ainsi que le parc automobile mondial s'est agrandi. Aujourd'hui, selon certains spécialistes, le nombre de véhicules en circulation devrait dépasser le milliard. Le continent Africain qui n'est pas producteur est submergé par des véhicules de tous types; et le phénomène est amplifié par l'importation des automobiles d'occasion. En Côte d'Ivoire, par exemple, ces véhicules de transport importés d'Europe sont appelés les «véhicules France au revoir ». Leur trafic occupe $65 \%$ des parts de marché de vente des véhicules en 2008 (Kablan, 2010). A Abidjan, pôle économique le plus important du pays, on dénombre environ 387.799 véhicules qui circulent quotidiennement. Ce chiffre représente plus de $82 \%$ du parc automobile de la Côte d'Ivoire estimé à 474.874 engins, selon le ministère de l'intérieur (Acturoute, 2016). Cette évolution fulgurante de l'effectif du parc automobile a un impact positif non négligeable sur l'économie du pays. Mais, elle a également des effets pervers sur l'environnement physique (la pollution avec les émissions de gaz à effet de serre, l'engorgement des voies) et humain (les accidents avec des pertes en vies humaines).

Pour illustrer ces conséquences négatives au plan humain dans le monde, l'Organisation Mondiale de la Santé (OMS) (2016) dénombre 1,25 million de décès environ par an dû aux accidents de la route, et $90 \%$ de ces décès proviennent de pays à revenu faible ou intermédiaire. L'OMS prévient en outre que ces accidents vont devenir la cinquième cause de décès dans le monde d'ici à 2030 si rien n'est fait.

En Côte d'Ivoire, le Ministère du Transport (2013) mentionne dans un rapport que les accidents de la circulation occasionnent en moyenne 600 décès et 13000 blessés par an. Ces statistiques établies en 2012 révèlent que $6 \%$ sont imputables aux autres facteurs (mauvais état des véhicules, du réseau routier, etc.) et $94 \%$ sont dus à des facteurs humains (conducteurs ou usagers).

La notion de facteur humain prend en compte l'ensemble des variables liées à la personne, et susceptibles d'avoir une incidence sur le comportement de conduite et sur l'occurrence d'accident (Elvik \& Vaa, 2004). D'après l'INSERM (2011), cette notion est imprécise, car elle renferme une multitude de variables d'ordre démographiques (âge ou le sexe) ; physiologiques (fatigue, les problèmes de santé, l'intoxication par l'alcool ou autres drogues); cognitives (inattention ou la distraction); comportementales (la prise de risque). 
Des travaux en psychologie ont porté sur la prise de risque dans la conduite automobile en mettant en évidence, par exemple, une forme de croyance appelée optimisme comparatif (Harris \& Middlton, 1994). Ils montrent que la plupart des automobilistes pensent qu'ils sont moins exposés que leurs pairs aux risques d'accidents (Belhaj \& Ghazali, 2014; Dejoy, 1989; Weinstein, 1980). Cette croyance est amplifiée par le contrôle subjectif (Delhomme, 2001). L'optimisme comparatif aurait des incidences sur la prise de risque, mais aussi sur la perception du risque dans la conduite automobile (Kouabenan, 1999). Lorsqu'un conducteur d'automobile évalue la probabilité que son comportement au volant entraîne des accidents, il perçoit le risque dans cette situation. Il donne son appréciation subjective de la possibilité que son action dans l'habitacle occasionne des dommages (Ulleberg \& Rundmo, 2003).

Dans le contexte ivoirien, l'observation du trafic routier montre que la prise de risque est un comportement qui caractérise les conducteurs, notamment les CPTC. On peut citer parmi leurs agissements les dépassements à droite, les circulations sur le trottoir, le non respect des feux tricolores et des distances de sécurité, etc. (Soro, 2015).

Pour remédier à ce problème, les gouvernants ivoiriens ont opté pour la sensibilisation des conducteurs et pour des mesures particulières dans les grandes villes comme Abidjan où le phénomène sévit avec acuité. A Abidjan, les moyens de déplacement en commun suivent des trajets précis déterminés par les autorités administratives et des campagnes sur la sécurité routière sont organisées à des périodes bien précises de l'année (à la veille des fêtes, en début et en fin d'année). Malgré ces mesures de sécurité et la multiplication des campagnes sur la prévention routière, les CPTC sont fréquemment impliqués et mis en cause dans la plupart des accidents ; principalement les plus jeunes et ceux qui ont le plus d'expérience dans la conduite, c'est-à-dire une plus longue durée de détention du permis et/ou plus d'années dans l'activité de transport en commun (Kouadio, 2013 ; 2015 ; Kouadio \& Méité, 2017). Ces différences entre conducteurs selon l'âge et l'expérience dans la conduite apparaissent également dans d'autres travaux (Groeger \& Brown, 1989 ; Harrington \& McBrid, 1970 ; McKnight \& Resnik, 1993).

Lors des constats d'accidents, les agents de police s'appuient très souvent sur des aspects techniques pour expliquer ces accidents. Or, les risques pris par ces CPTC lors de leurs activités de transport pourraient être à la base de la majeure partie des accidents. Dans les tentatives d'explication, on suggère même d'orienter les réflexions vers les facteurs cognitifs, notamment la perception du risque (Kouabenan, 2007). En Côte d'Ivoire, les travaux qui ont examiné les risques routiers sont rares semble-t-il. C'est pourquoi, la présente étude examine la perception du risque d'accidents dans 
la conduite automobile pour tenter de comprendre les nombreuses infractions routières observées chez les CPTC dans le contexte ivoirien.

Quelle est donc la situation de la perception du risque d'accidents liés à la conduite des CPTC à Abidjan ?

La présente étude se propose d'évaluer la perception du risque d'accidents liés à la conduite des CPTC à Abidjan. Ainsi, les hypothèses qui suivent sont formulées. H1 : Le nombre de CPTC qui perçoivent le moins le risque d'accidents liés à la conduite est supérieur à celui de leurs pairs qui le perçoivent le plus. H2 : L'âge et la durée de détention du permis de conduire influencent conjointement la perception du risque d'accidents liés à la conduite des CPTC.

\section{Méthodologie \\ Participants}

L'enquête a concerné 186 Conducteurs Professionnels de Transport en Commun d'Abidjan dont l'âge varie entre 18 et 50 ans. Ces participants ont une durée de détention du permis de conduire différente (0-2 ans ; 3-5 ans et plus de 5 ans).

\section{Instruments}

42 conducteurs professionnels tout venant de la ville d'Abidjan ont été invités, dans un entretien, à citer trois manœuvres et trois comportements liés à l'activité de conduite automobile susceptibles de causer des accidents de la circulation. Les données fournies par ces conducteurs ont permis de construire un questionnaire de perception du risque d'accidents. Outre, les items relatifs aux caractéristiques des participants (âge, année d'obtention du permis de conduire), ce questionnaire comporte 37 items sur une échelle à 5 points allant de 1 (pas du tout d'accord) à 5 (tout à fait d'accord). Voici des exemples : « ne pas faire attention aux autres usagers de la circulation n'entraine pas d'accident »; « les causeries au volant n'entrainent pas d'accident »; « rouler sans clignotants provoque des accidents sur les routes $»$.

La moyenne des scores de la perception est calculée pour classer les conducteurs selon que leur perception est bonne ou mauvaise. Tous les participants qui ont alors un score en dessous du score moyen à l'échelle globale $(\mathrm{M}=2,30)$ sont considérés comme ayant une bonne perception, tandis que ceux qui ont un score au-dessus de la valeur moyenne sont considérés comme ayant une mauvaise perception.

\section{Procédure}

Une sélection des personnes à contacter s'est faite par le biais d'entretien semi-directif. Les critères d'hétérogénéité ont prévalu en vue de prendre en compte une diversité de participants. Ils sont tous des volontaires 
et ont été rencontrés individuellement à travers les rues de la ville, sur certains lieux de travail, dans les gares automobiles et dans des espaces publics.

\section{Résultats}

Les résultats de l'analyse statistique obtenus à partir du logiciel SPSS 17.0 sont présentés en deux axes. Le premier prend en compte les résultats de l'analyse factorielle des items du questionnaire de perception du risque routier. Le second axe composé de deux points, vérifie les hypothèses formulées.

\section{Analyse factorielle des items de la perception de risque routier}

L'analyse factorielle en composante principale (ACP) avec rotation Varimax met en évidence 7 facteurs expliquant $61,65 \%$ de la variance totale. Cependant, ce sont les trois premiers facteurs qui ont été retenus sur la base du graphique des valeurs propres.

Les facteurs retenus désignent en quelque sorte les principales variables contribuant aux risques encourus par les conducteurs dans leurs activités de transport. Il s'agit de la stabilité émotionnelle et la posture au volant ( $1^{\text {er }}$ facteur); de l'interaction avec autrui et l'état de fatigue ( $2^{\text {ième }}$ facteur); de l'état du véhicule et la conscience au volant ( $3^{\text {ième }}$ facteur) (tableau 1).Ces facteurs sont tous composites.

L'activité de transport en commun n'est pas une activité de tout repos. La stabilité émotionnelle et la posture au volant des conducteurs constituent un véritable problème. Les conducteurs sont soumis à des influences externes (exigences des passagers, autres usagers de la route, etc.) qui les déstabilisent sur le plan émotionnel; et c'est généralement dans cet état qu'ils conduisent leur véhicule et négligent les autres aspects comme la posture au volant. Un autre problème qui affecte négativement le secteur du transport en commun en Côte d'Ivoire est l'état des véhicules. Les conducteurs de transport en commun rencontrés à Abidjan sont majoritairement des analphabètes ou du niveau primaire et n'ont bénéficié d'aucune autre formation à part celle du permis de conduire obtenu une auto-école. Ainsi, l'approche client leur fait énormément défaut. Ils ont aussi un problème de gestion du temps de travail qui agit amplement sur leur état de fatigue. Enfin, la plupart des automobiles utilisées pour le transport en commun manque d'entretien, et présente des aspects très dégradés. Leurs utilisateurs semblent ne pas en prendre conscience, mettant ainsi les passagers et les autres usagers en danger perpétuel. Cet état d'inconscience transparait également dans le style de conduite qui est le plus souvent porté vers le viol flagrant et permanent des règles élémentaires du code de la route. 
Tableau 1 : Les facteurs conduisant aux accidents

\begin{tabular}{|c|c|c|}
\hline Facteurs & Items & Alpha de cronbach \\
\hline $\begin{array}{l}\text { Stabilité émotionnelle et } \\
\text { posture au volant }\end{array}$ & $\begin{array}{l}\text {-Q14 : le manque de sérénité au volant } \\
\text { n'entraine pas d'accidents } \\
\text {-Q21 : l'impatience n'entraine pas } \\
\text { d'accidents de la circulation } \\
\text {-Q36 : ne pas ajuster le siège de } \\
\text { conduite ne conduit pas à des } \\
\text { accidents de la circulation } \\
\end{array}$ & 0,60 \\
\hline $\begin{array}{l}\text { Interaction avec autrui et état } \\
\text { de fatigue }\end{array}$ & $\begin{array}{c}\text {-Q26 : conduire lorsqu'on est fatigué } \\
\text { n'occasionne pas d'accidents } \\
\text {-Q33 : trop écouter les consignes } \\
\text { (amis, apprentis) ne conduit pas aux } \\
\text { accidents } \\
\text {-Q34 : prendre un ami ou un client en } \\
\text { pleine chaussée n'occasionne pas } \\
\text { d'accidents } \\
\text {-Q23 : la mauvaise humeur au volant } \\
\text { n'entraine pas d'accident de la } \\
\text { circulation } \\
\end{array}$ & 0,61 \\
\hline $\begin{array}{c}\text { Etat du véhicule et conscience } \\
\text { au volant }\end{array}$ & $\begin{array}{c}\text {-Q35 : la surcharge du véhicule cause } \\
\text { des accidents sur les routes } \\
\text {-Q28 : les causeries au volant } \\
\text { n'entraînent pas des accidents de la } \\
\text { circulation } \\
\text {-Q30 : fumer au volant occasionne des } \\
\text { accidents de la circulation } \\
\end{array}$ & 0,51 \\
\hline
\end{tabular}

\section{La perception du risque d'accidents sur l'ensemble de l'échantillon}

Tableau 2 : Répartition des conducteurs selon leur perception

\begin{tabular}{cccc}
\hline & Effectif observé & Effectif théorique & Résidu \\
\hline Mauvaise perception & 98 & 93,0 & 5,0 \\
\hline Bonne perception & 88 & 93,0 & $-5,0$ \\
\hline Total & 186 & &
\end{tabular}

L'hypothèse H1 n'est pas confirmée. Il existe autant de CPTC qui perçoivent le moins les risques d'accidents liés à la conduite que ceux qui le perçoivent le plus $\left(\chi^{2}=0,54, \mathrm{p}>.05\right)$.

\section{Perception du risque d'accidents et caractéristiques individuelles}

Les caractéristiques individuelles considérées ici sont l'âge (moins de 30 ans vs 30 ans et plus) et la durée de détention du permis de conduire (0-2 ans ; 3-5 ans ; plus de 5 ans) des CPTC.

-Age et perception du risque d'accidents

Tableau 3 : Comparaison des moyennes de perception du risque

\begin{tabular}{cccccc}
\hline & & $\mathrm{N}$ & $\mathrm{M}$ & $\mathrm{ET}$ & ESM \\
\hline \multirow{3}{*}{ Age } & moins de 30 ans & 39 & 2,14 &, 56 &, 09 \\
\cline { 2 - 6 } & 30 ans et plus & 147 & 2,34 &, 44 &, 04 \\
\hline
\end{tabular}


La différence entre les tranches d'âges est statistiquement significative $(\mathrm{t}=2,31, \mathrm{p}<.05)$. L'hypothèse $\mathrm{H} 2$ se confirme si on tient compte de l'âge des conducteurs. On en déduit que les conducteurs jeunes, c'est-à-dire ceux de moins de 30 ans perçoivent le plus les risques d'accidents comparativement à leurs homologues de 30 ans et plus.

-Durée de détention du permis de conduire (PC) et perception du risque d'accidents

Tableau 4 : Les moyennes selon la durée de détention PC

\begin{tabular}{llll} 
& & $\mathrm{N}$ & $\mathrm{M}$ \\
\hline \multirow{3}{*}{ Durée de détention du PC } & $0-2$ ans & 25 & 1,99 \\
\cline { 2 - 4 } & $3-5$ ans & 26 & 2,40 \\
\cline { 2 - 4 } & plus de 5 ans & 135 & 2,33 \\
\hline
\end{tabular}

Tableau 5 : Analyse de variance (ANOVA)

\begin{tabular}{cccccc}
\hline & $\begin{array}{c}\text { Somme des } \\
\text { carrés }\end{array}$ & ddl & $\begin{array}{c}\text { Moyenne des } \\
\text { carrés }\end{array}$ & F & Sig. \\
\hline Inter-groupes & 2,743 & 2 & 1,372 & 6,518 &, 002 \\
\hline Intra-groupes & 38,515 & 183 &, 210 & & \\
\hline Total & 41,258 & 185 & & & \\
\hline
\end{tabular}

L'ANOVA révèle que la perception du risque d'accidents des conducteurs varie significativement en fonction de la durée de détention du permis de conduire $(\mathrm{F}=6,52, \mathrm{p}<.05)$. L'hypothèse $\mathrm{H} 2$ est alors confirmée si la durée de détention du permis de conduire est prise en compte. Les comparaisons deux à deux au test de tukey indique que les conducteurs qui ont entre 0-2 ans de durée de détention du PC perçoivent plus les risques d'accidents que ceux de 3-5 ans et de plus de 5 ans de durée de détention du PC. La différence entre ceux de 3-5 ans et ceux de plus de 5 ans de durée de détention du PC n'est pas prononcée.

-Effet d'interaction de l'âge et de la durée de détention du permis de conduire (PC) sur la perception du risque d'accidents

Tableau 6 : Tests des effets inter-sujets

Somme des

carrés de type

\begin{tabular}{cccccc} 
Source & III & ddl & carrés & F & p \\
\hline Modèle corrigé & $3,171(\mathrm{a})$ & 5 &, 634 & 2,997 &, 013 \\
\hline Constante & 241,154 & 1 & 241,154 & 1139,688 &, 000 \\
\hline Age &, 001 & 1 &, 001 &, 003 &, 954 \\
\hline DPC & 1,946 & 2 &, 973 & 4,598 &, 011 \\
\hline age * DPC & $\mathbf{, 3 8 7}$ & $\mathbf{2}$ & $\mathbf{, 1 9 4}$ & $\mathbf{9 1 5}$ & $\mathbf{4 0 2}$ \\
\hline Erreur & 38,087 & 180 &, 212 & & \\
\hline Total & 1024,424 & 186 & & & \\
\hline Total corrigé & 41,258 & 185 &
\end{tabular}


L'effet conjoint de ces deux variables sur la perception du risque d'accidents n'est pas significatif $(F=0,91, p>.05)$. L'hypothèse $H 2$ n'est pas confirmée si l'âge et le PC sont pris simultanément en compte. Il n'y a donc pas d'effet d'interaction entre l'âge des conducteurs et la durée de détention de leur PC sur la perception qu'ils ont du risque d'accidents.

Il ressort de ce travail que la perception du risque d'accidents des CPTC ivoiriens s'articule autour de trois composantes principales : la stabilité émotionnelle et la posture au volant, l'interaction avec autrui et l'état de fatigue, et l'état du véhicule et la conscience au volant. C'est autour de ces composantes que sont cristallisées les perceptions des conducteurs. C'est aussi ces composantes qui sont à l'origine des accidents de la route chez les CPTC.

L'étude fait observer que l'écart entre les CPTC qui perçoivent le moins les risques d'accidents et ceux qui les perçoivent le plus n'est pas significatif. Cette tendance est peut être due à la non prise en compte dans l'étude de l'expérience dans le métier de CPTC qui a été assimilée à la durée de détention du PC. Or, ces deux périodes, qui peuvent coïncider chez certains conducteurs, sont deux réalités différentes.

En outre, deux caractéristiques (l'âge et la durée de détention du permis de conduire) influencent individuellement la perception du risque d'accidents de ces conducteurs. Concrètement, ce sont les plus jeunes et ceux qui ont une durée de détention du permis de conduire plus courte qui perçoivent le plus le risque d'accidents.

Enfin, l'effet d'interaction entre ces variables n'est pas statistiquement significatif au point de créer des différences entre les conducteurs quant à leur perception du risque.

\section{Discussion}

Ces résultats qui viennent d'être évoqués sont en accord avec certains aspects des travaux déjà réalisés en Côte d'Ivoire (Kouadio, 2013 ; 2015 ; Kouadio \& Méité, 2017).

D'abord concernant l'âge, ce sont les conducteurs les plus jeunes qui perçoivent le plus les risques d'accidents comparativement aux plus âgés. Ce résultat semble surprenant parce qu'il ne s'accorde pas avec les idées reçues et les observations faites par le sens commun. Une partie de l'explication de la différence entre ces deux groupes peut provenir du phénomène de l'optimisme comparatif (Harris \& Middlton, 1994). En effet, les conducteurs plus âgés penseraient que leur maturité et leurs expériences dans la vie leur confèrent un aguerrissement pour tout type d'activité, en l'occurrence l'activité de conduite de transport en commun. De ce fait, ils seraient moins exposés aux risques d'accidents que leurs homologues plus jeunes ; ce qui les amènerait à avoir une faible perception de risques d'accidents et à croire que l'activité de conduite de transport en commun serait une activité sans danger. 
Cette croyance serait contraire à celle des jeunes. De plus, un conducteur peut avoir un âge avancé mais peut être novice dans l'activité de transport en commun. Le métier de conducteur professionnel de transport en commun en Côte d'Ivoire n'est pas un secteur facile d'accès vu le coût d'acquisition d'un permis de conduire et l'achat d'un véhicule homologué au transport en commun. Alors, malgré leur âge avancé, certaines personnes peinent à avoir les moyens financiers pour exercer ce métier. Ainsi, il n'est pas exclu qu'on rencontre très souvent des conducteurs plus âgés, mais inexpérimentés dans la conduite.

La différence entre jeunes conducteurs et conducteurs plus âgés est mentionnée également dans l'étude de Harrington \& McBrid (1970). Elle montre que le type d'infraction routière varierait en fonction de la classe d'âge à laquelle appartient le conducteur, mais n'écarte pas la possibilité que les conducteurs adultes obtiennent un nombre important de violation de règles de conduite que les conducteurs jeunes.

Concernant la durée de détention du permis de conduire, l'étude révèle que ce sont les conducteurs qui comptabilisent le plus d'années qui perçoivent le moins les risques d'accidents. Ici également, l'optimisme comparatif peut être invoqué pour comprendre cette tendance. Ceux qui ont plus d'ancienneté au regard de la durée de leur permis de conduire croient probablement qu'ils seront moins portés à commettre des accidents que leurs pairs novices. Ils ont un contrôle subjectif sur l'activité de conduite (Delhomme, 2001). Or, comme il a été précédemment évoqué, la durée de détention du PC peut ne pas correspondre à l'ancienneté dans le métier de conducteur de transport en commun. Dans le contexte ivoirien, notamment à Abidjan, la conduite d'un véhicule de transport en commun éprouve profondément le conducteur professionnel. C'est pourquoi, en plus des compétences de conduite, le conducteur doit avoir d'autres capacités dans la gestion des clients, des émotions, du temps de travail, et la connaissance de la cartographie de la ville. Ce résultat s'accorde avec celui de McKnight et Resnik (1993), mais contredit les observations de Groeger et Brown (1989) qui précisent que l'inexpérience se traduit par une plus grande difficulté à identifier les risques plus tôt et d'y répondre convenablement.

En somme, dans le contexte ivoirien, la perception du risque peut aussi être envisagée dans l'explication des accidents routiers. Car, dans cette composante psychologique, les conducteurs présentent des différences interindividuelles sous l'angle de l'âge et de la durée de détention du permis de conduire.

\section{Conclusion}

La diminution du nombre d'accidents et des victimes sur les routes ne pourra probablement se faire que s'il y a une réelle prise de conscience des 
conducteurs du danger que peut constituer la conduite automobile ; mais aussi si l'on élimine un certains nombre de facteurs de risques d'accidents. La réussite de ces actions devrait être appuyée principalement par une véritable volonté politique de prise en charge de la sécurité routière.

Cependant, au regard de l'importance des facteurs humains dans les accidents en Côte d'Ivoire, il est plus qu'urgent d'axer les sensibilisations sur des aspects inhérents à l'individu. Dans ce contexte, la présente étude pourrait aider au diagnostic de la prise de risques dans la conduite automobile chez des individus en suggérant un examen psychologique consécutif à l'examen médical (groupage et acuité visuelle). Dans le circuit de délivrance du permis de conduire, ce dispositif évaluera la tendance du candidat à la prise de risques dans l'activité de conduite automobile et sa perception. C'est une étape au cours de laquelle le candidat pourrait passer des échelles ou des tests appuyés d'entretiens semi-directifs sur la prise de risques dans la conduite. C'est une phase qui pourrait déboucher sur des campagnes de sensibilisation routières ciblées. L'étude pourrait aussi servir de base pour la formalisation d'un modèle d'accompagnement à la conduite automobile des candidats au permis de conduire et des professionnels du transport.

\section{References:}

1. Acturoute (2016). Abidjan : plus de 300.000 véhicules en circulation. Extrait de : https://www.acturoutes.info/page.php?p=1\&id=4279

2. Belhaj A. \& Ghazali A. (2014). Optimisme comparatif et comportement préventif chez les conducteurs professionnels des grands véhicules routiers. In R. Ngueutsa, R. Mokounkolo, N. Achi, \& A. Belhaj, (Sous la direction), Psychologie du travail et développement des pays du Sud (p. 229-160). Paris : L'Harmattan.

3. Dejoy D.M. (1989). The optimism bias and traffic accident risk perception. Accident Analyis and Prevention, 21, 333-340.

4. Delhomme P. (2001). Évaluation d'actions possibles face à un risque : une approche expérimentale de l'effet du contrôle absolu et comparatif. Revue Internationale de psychologie sociale, 14, 45-81.

5. Elvik R. \& Vaa T. (2004). The handbook of road safety measures. Oxford : Elsevier Science.

6. Groeger J.A. \& Brown I.D. (1989). Assessing one's own and others's driving ability: influences of sex, age, and experience. Accident Analysis and Prevention, 21(2), 155-168.

7. Harrington D.M. \& McBrid R.S. (1970). Traffic violation by type, age, sex and marital status. Accident Analysis and Prevention, 1, 9-16.

8. Harris P. \& Middleton W. (1994). The illusion of control and optimism about health : On being less at risk but no more in control than others. British Journal of Social Psychology, 33, 369-386. 
9. Inserm (2011). Téléphone et sécurité routière. Paris : Les éditions Inserm.

10. Kablan N.H.J. (2010). L'invasion des véhicules d'occasion en transite par le port d'Abidjan : Le dynamisme ambivalent d'une activité en plein essor. Les Cahiers d'Outre-Mer, 151, 365-390.

11. Kouabenan D.R. (1999). Explication naïve de l'accident et prévention. Paris : Presses Universitaires de France.

12. Kouabenan D.R. (2007). Incertitude, croyances et management de la sécurité. Le Travail Humain, 70(3), 271-287.

13. Kouadio K.A. (2013). Activités de transport en commun, âge et comportements de conduite automobile chez des conducteurs de taxicompteur et gbaka à Abidjan. Cahiers Ivoiriens de Psychologie, 3, 99115.

14. Kouadio K.A. (2015). Psychologie des conducteurs de véhicule de transport en commun: Aspects comportementaux des conducteurs jeunes et adultes de taxi-compteur, woro et gbaka à Abidjan. Saarbrücken : Editions Universitaires Européennes.

15. Kouadio K.A. \& Méité A. (2017). Accidents de la route et comportements de conduite chez des conducteurs professionnels ivoiriens. In S. Gaymard et T. Tiplica (Eds), Sécurité routière : un défit à l'aube du XXe siècle (pp 81-87). Paris : L'Harmattan.

16. McKnight A. \& Resnik J. (1993). Youthful driver at risk, background paper. National Public Service Research Institue, US DOT, 27 avril 1993.

17. Ministère du Transport (novembre 2013). Transport Info : La sécurité routière une responsabilité sociétale. $2^{\text {ième }}$ Edition de la journée Africaine de la sécurité routière.

18. Organisation Mondiale de la Santé (2016). Accidents de la route. Aidemémoire novembre 2016. Extrait de : http://www.who.int/mediacentre/factsheets/fs358/fr/

19. Soro, K. (2015). Perception des risques d'accidents associés à la conduite imprudente: Le cas des conducteurs de la ville d'Abidjan. Mémoire de Master Conseiller Psychologue, CIERPA-Université Félix Houphouët Boigny.

20. Ulleberg P. \& Rundmo T. (2003). Personality, attitudes and risk perception as predictors of risk driving behaviour among young drivers. Safety Science, 41, 427-443.

21. Weinstein N.D. (1980). Unrealistic optimism about future life events. Journal of Personality and Social Psychologie, 39(5), 806-820. 